\title{
Crescimento e produção de plantios comerciais de eucalipto estimados por duas categorias de modelos
}

\author{
Renato Vinícius Oliveira Castro(1), Carlos Pedro Boechat Soares ${ }^{(2)}$, \\ Fabrina Bolzan Martins ${ }^{(3)}$ e Helio Garcia Leite ${ }^{(2)}$
}

\begin{abstract}
(1)Universidade de Brasília, Departamento de Engenharia Florestal, Campus Darci Ribeiro, CEP $70904-970$ Brasília, DF. E-mail: castrovo@ymail.com (2)Universidade Federal de Viçosa, Departamento de Engenharia Florestal, Avenida P.H. Rolfs, s/no, Campus Universitário, CEP 36570-000 Viçosa, MG. E-mail: csoares@ufv.br, hgleite@gmail.com (3)Universidade Federal de Itajubá, Instituto de Recursos Naturais, Avenida BPS, no 1.303, Bairro Pinheirinho, CEP 37500-903 Itajubá, MG. E-mail: fabrinabm@gmail.com
\end{abstract}

\begin{abstract}
Resumo - O objetivo deste trabalho foi avaliar e comparar duas categorias de modelos de crescimento e produção em plantios comerciais de eucalipto. Para isso, foram ajustados um modelo de crescimento e produção para povoamento e outro para árvore individual, por meio de equações simultâneas e redes neurais artificiais, respectivamente. $\mathrm{O}$ volume de madeira por área foi estimado em diferentes idades e classes de produtividade. Foram avaliados dados de 63 parcelas permanentes de plantios clonais, não desbastados, do híbrido Eucalyptus grandis $\mathrm{x}$ E. urophylla, com os dados de 33 parcelas utilizados para o ajuste do modelo e o treinamento das redes neurais, e os das 30 parcelas restantes, para a validação dos modelos. As duas categorias de modelos ajustaram-se bem aos dados observados. No entanto, na validação dos modelos com dados independentes, o volume de madeira por área foi mais bem estimado com o modelo para árvore individual.
\end{abstract}

Termos para indexação: Eucalyptus, equações simultâneas, modelagem do crescimento, produção, redes neurais artificiais.

\section{Growth and yield of commercial plantations of eucalyptus estimated by two categories of models}

\begin{abstract}
The objective of this work was to evaluate and compare two categories of growth and yield models of commercial plantations of eucalyptus. For that, a whole stand model, and an individual tree growth model were adjusted through simultaneous equations and artificial neural networks, respectively. Wood volume per area was estimated for tree different ages and productivity classes. Data of 63 permanent plots of unthinned clonal hybrid Eucalyptus grandis x E. urophylla were evaluated, from which those referring to 33 plots were used for model fitting and neural network training, and those referring to the other 30 plots were used for model validation. The two categories of models adjusted well to the observed data. However, for the validation of the models with independent data, the wood volume per area was better estimated with the individual tree growth model.
\end{abstract}

Index terms: Eucalyptus, simultaneous equations, growth modeling, yield, artificial neural networks.

\section{Introdução}

Os modelos de crescimento e produção florestal permitem simular a dinâmica natural de um povoamento e prever a sua produção ao longo do tempo, em diferentes possibilidades de exploração (Vanclay, 1994). Na área florestal, as três principais categorias de modelos de crescimento e produção são direcionadas para povoamento, distribuição diamétrica e para árvores individuais (Davis et al., 2005).

A categoria para povoamentos totais (MPT) fornece estimativas do crescimento ou da produção por unidade de área (Campos \& Leite, 2009), e o volume por área predito ou projetado é obtido a partir de variáveis como idade do povoamento, índice de local e área basal (Davis et al., 2005). Essa categoria de modelo não fornece estimativas do tamanho das árvores, o que, no entanto, não limita o seu emprego quando o manejo é destinado à produção de celulose ou energia (Campos \& Leite, 2009).

Já a categoria para distribuição diamétrica (MDD) estima o número de árvores e a produção por hectare por classe de diâmetro, e a produção total é obtida por meio do somatório da variável de interesse em todas as classes de diâmetro (Campos \& Leite, 2009). Nesta categoria, empregam-se funções de densidade 
de probabilidade, para descrever a distribuição dos diâmetros na idade atual e futura, em classes de amplitudes previamente fixadas, das quais, a função de Weibull é a mais utilizada (Miguel et al., 2010). A prognose da produção volumétrica é realizada por modelos de regressão, utilizados na forma de sistemas de equações que estimam os parâmetros da função para idades futuras. A partir desta distribuição, pode ser estimado o número de árvores por classe de diâmetro e a produção volumétrica.

A categoria para árvores individuais (MAI) é mais complexa do que as demais; porém, ela tem a vantagem de gerar informações detalhadas sobre a dinâmica da estrutura dos povoamentos. Neste modelo, cada árvore é estudada individualmente, levando-se em conta suas características individuais, a capacidade produtiva do local e as condições de competição às quais a árvore está submetida (Hasenauer et al., 2006). O MAI é constituído por um conjunto de submodelos que estimam ingresso e probabilidade de mortalidade, além do crescimento em diâmetro e altura (Davis et al., 2005; Campos \& Leite, 2009).

Independentemente da categoria, os modelos de crescimento e produção baseiam-se no ajuste de modelos de regressão linear ou não linear. No entanto, ferramentas de inteligência artificial, como as redes neurais artificiais (RNA), têm sido empregadas com sucesso na área florestal (Görgens et al., 2007; Silva et al., 2009; Castro, 2011), em substituição aos tradicionais modelos de regressão, principalmente em relação ao MAI que, muitas vezes, é inviável em razão da grande quantidade de submodelos que o compõe.

O MPT atende de modo eficiente à maioria dos usuários e, atualmente, é a categoria mais utilizada pelas empresas florestais do Brasil. No país, o modelo de Clutter vem sendo amplamente utilizado desde meados da década de 80 , em razão de sua menor complexidade e pelo fato de atender às exigências de planejamento para produção de madeira de uso único (celulose ou carvão vegetal), principalmente em plantios de eucalipto e pinus (Leite et al., 2001; Dias et al., 2005; Görgens et al., 2007). No entanto, muitas vezes, o detalhamento das informações acerca do crescimento e produção dos povoamentos é necessário, principalmente para estimar a produção de madeira de alta qualidade e a de madeira destinada a múltiplos usos. Assim, o modelo para árvore individual, apesar de pouco utilizado no Brasil (Martins, 2011; Martins et al., 2011), apresenta uso bem mais expressivo na Europa, EUA, Canadá, África e Austrália
(Mabvurira \& Miina, 2002; González et al., 2006; Monty et al., 2008; Vospernik et al., 2010). O menor uso desses modelos no Brasil está relacionado à falta de dados apropriados para o seu ajuste, à sua complexidade, ao maior número de funções que compõem o modelo e à falta de estudos que demonstrem sua precisão.

O objetivo deste trabalho foi avaliar e comparar duas categorias de modelos de crescimento e produção em plantios comerciais de eucalipto.

\section{Material e Métodos}

Os dados utilizados foram obtidos de inventários florestais contínuos, entre 1997 e 2003, de povoamentos clonais híbridos não desbastados de Eucalyptus grandis W. Hill ex Maiden x E. urophylla S.T. Blake (Tabela 1), localizados na cidade de Monte Dourado, PA $\left(0^{\circ} 53^{\prime} 22^{\prime \prime} \mathrm{S}\right.$, $\left.52^{\circ} 36^{\prime} 6^{\prime \prime} \mathrm{W}\right)$. O clima da região, segundo a classificação de Köppen, é do tipo Am, com precipitação média anual de $2.115 \mathrm{~mm}$, temperatura média anual de $26^{\circ} \mathrm{C}$, e umidade relativa entre 80 e $85 \%$ (Martins et al., 2011).

Foram utilizados dados de 63 parcelas permanentes, localizadas em seis diferentes locais de plantio. Cada parcela teve formato retangular, com $500 \mathrm{~m}^{2}$ de área útil, e o espaçamento entre plantas foi de $3 \times 3 \mathrm{~m}$. Em cada parcela, foram realizadas cinco medições, tendo-se considerado três classes de produtividade (alta, média e baixa).

Foram mensurados: os diâmetros com casca à altura $(1,30 \mathrm{~m})$ do peito (dap, $\mathrm{cm})$ em todas as árvores com dap maior que 4,0 cm; as alturas totais (h, m) das 15 primeiras árvores; e a altura total das cinco árvores dominantes (hd), conforme Assmann (1970). Para estimar a altura total das demais árvores da parcela, foi utilizada a equação hipsométrica, ajustada por

Tabela 1. Dados do povoamento de Eucalyptus grandis $\mathrm{x}$ E. urophylla utilizado no estudo, referentes ao período 1997-2003.

\begin{tabular}{lcc}
\hline Parâmetro & \multicolumn{2}{c}{ Idade (meses) $^{(1)}$} \\
\cline { 2 - 3 } & 24 & 72 \\
\hline Diâmetro à altura do peito (cm) & 4,0 & 29,4 \\
Diâmetro médio (cm) & 7,3 & 18,4 \\
Altura total (m) & 8,5 & 34,1 \\
Altura dominante (m) & 13,1 & 34,8 \\
Área basal $\left(\mathrm{m}^{2} \mathrm{ha}^{-1}\right)$ & 4,7 & 27,2 \\
Volume $\left(\mathrm{m}^{3}\right.$ ha $\left.^{-1}\right)$ & 23,8 & 353,9 \\
Densidade de árvores (árvores ha-1) & 760 & 1.180 \\
(1) Valor mínimo e máximo do povoamento aos 24 & e 72 meses, \\
respectivamente. & &
\end{tabular}


Demolinari (2006) à área de estudo, abaixo representada: $\mathrm{h}=36,9876-30,4340 \exp \{-0,000499$ [dap $\times \ln$ hd $\left.\times \ln \mathrm{I}^{1,388275}\right\}$, com $\mathrm{R}^{2}=83,7 \%$ e $\mathrm{S}_{\mathrm{y}, \mathrm{x}}= \pm 11,79 \%$, em que: I é a idade, em meses; e $S_{y . x}$ é o erro-padrão da estimativa.

As parcelas permanentes estavam localizadas em seis locais diferentes. Para cada local, foi utilizada uma equação específica, para determinar o volume de madeira com casca das árvores (Tabela 2) (Demolinari, 2006).

Para a classificação da classe de produtividade, foi utilizada a equação de Schumacher, ajustada para a mesma área de estudo (Demolinari, 2006): $\ln \mathrm{S}=\ln \mathrm{hd}+14,88011596\left(1 / \mathrm{I}_{\mathrm{i}}+1 / \mathrm{I}\right)$, em que: $\mathrm{S}$ é o índice de local, adimensional; e $\mathrm{I}_{\mathrm{i}}$ é a idade-índice (60 meses).

Os limites para a classificação das parcelas, nas classes de produtividade, foram: classe de produtividade alta $(\mathrm{S}=32)$, parcelas com $\mathrm{hd}>29 \mathrm{~m}$ na idade-índice de 60 meses; classe de produtividade média $(\mathrm{S}=26)$, parcelas com hd entre 24 e $29 \mathrm{~m}$; classe de produtividade baixa $(\mathrm{S}=20)$, parcelas com $\mathrm{hd}<24 \mathrm{~m}$, na idade-índice de 60 meses.

O conjunto de parcelas permanentes foi dividido aleatoriamente em dois grupos. O primeiro grupo, composto de 33 parcelas (11 em cada classe de produtividade), foi utilizado para o ajuste das equações do MPT e treinamento das redes neurais artificiais do MAI. Nas cinco medições realizadas, este grupo totalizou 8.735 medidas de árvores individuais, com média de 53 árvores por parcela. $\mathrm{O}$ segundo grupo, composto pelas 30 parcelas restantes (10 em cada classe de produtividade), foi utilizado para a validação das duas categorias de modelos (MPT e MAI), com 7.756 medições.

O modelo para o povoamento total utilizado no presente trabalho foi o de Clutter (1963), composto pelo sistema de equações simultâneas:

$\ln \mathrm{B}_{2}=\ln \mathrm{B}_{1}\left(\mathrm{I}_{1} / \mathrm{I}_{2}\right)+\alpha_{1}\left(1-\mathrm{I}_{1} / \mathrm{I}_{2}\right)+\alpha_{2}\left(1-\mathrm{I}_{1} / \mathrm{I}_{2}\right) \times \mathrm{S}+\varepsilon$

$\ln \mathrm{V}_{2}=\beta_{1}+\beta_{2}\left(1 / \mathrm{I}_{2}\right)+\beta_{3} \mathrm{~S}+\beta_{4} \times \ln \mathrm{B}_{2}+\varepsilon$

Tabela 2. Equações utilizadas para predição do volume de madeira com casca.

\begin{tabular}{ll}
\hline Área & Equação \\
\hline 001 & $\ln \mathrm{V}=-11,145922+1,886699 \ln$ dap $+1,3733330 \ln \mathrm{h}$ \\
016 & $\ln \mathrm{V}=-9,850475+1,7856259 \ln$ dap $+1,0923825 \ln \mathrm{h}$ \\
026 & $\ln \mathrm{V}=-10,162655+1,8427101 \ln \mathrm{dap}+1,1041629 \ln \mathrm{h}$ \\
041 & $\ln \mathrm{V}=-10,636733+1,9185875 \ln \mathrm{dap}+1,2004476 \ln \mathrm{h}$ \\
042 & $\ln \mathrm{V}=-10,508245+1,8839540 \ln$ dap $+1,1755465 \ln \mathrm{h}$ \\
077 & $\operatorname{lnV}=-10,382205+1,8935363 \ln$ dap $+1,1283936 \ln \mathrm{h}$ \\
\hline
\end{tabular}

$\mathrm{V}$, volume de madeira com casca das árvores, $\mathrm{em} \mathrm{m}^{3}$; dap, diâmetro à altura do peito $(1,30 \mathrm{~m}), \mathrm{em} \mathrm{cm}$; $\mathrm{h}$, altura total, em $\mathrm{m}$. em que: $\mathrm{B}_{2}$ é a área basal do povoamento na idade futura $\left(\mathrm{m}^{2} \mathrm{ha}^{-1}\right) ; \mathrm{B}_{1}$ é a área basal do povoamento na idade atual; $\mathrm{I}_{1}$ é a idade atual, em meses; $\mathrm{I}_{2}$, a idade futura; $\mathrm{S}$ é o índice de local; $\mathrm{V}_{2}$ é o volume de madeira com casca do povoamento, na idade futura $\left(\mathrm{m}^{3} \mathrm{ha}^{-1}\right)$; com $\alpha_{1}$ e $\alpha_{2}$, que são os coeficientes do modelo de área basal; $\beta_{1} \ldots \beta_{4}$ são os coeficientes do modelo volumétrico; e $\varepsilon$ é o erro aleatório.

As estimativas dos parâmetros do MPT, com base nas equações simultâneas de Clutter (1963), foram obtidas com o método dos mínimos quadrados, em dois estágios, com auxílio do programa Eviews 5.0 (IHS Global, 2004).

Os ajustes das equações foram avaliados por meio do coeficiente de correlação e do erro-padrão da estimativa $\left(\mathrm{S}_{\mathrm{y} \mathrm{x}}\right)$ (Leite et al., 2011). Além dessas estatísticas, a análise do erro em percentagem e as análises gráficas da área basal e do volume observado foram realizadas em relação aos seus valores estimados.

No MAI foi realizado o treinamento das redes neurais artificiais, por meio da ferramenta "intelligent problem solver", do programa Statistica 7.0, e as diferentes arquiteturas de redes Multilayer Perceptron (MLP) foram testadas. As redes MLP são redes multicamadas "feedforward", que possuem camadas ocultas entre as camadas de entrada e saída. As camadas ocultas conseguem extrair padrões não lineares dos dados, e uma rede MLP, com duas camadas intermediárias, permite a aproximação de qualquer função (Haykin, 2001).

Os treinamentos da rede MLP foram do tipo "feedforward", pelo método supervisionado. Este tipo utiliza algoritmo de fluxo de dados em uma única direção, sem ciclos, enquanto no método supervisionado, as variáveis de entrada e saída são indicadas para o treinamento (Haykin, 2001).

As variáveis de entrada do modelo para árvore individual foram: idade atual e futura $\left(\mathrm{I}_{1}\right.$ e $\left.\mathrm{I}_{2}\right)$, índice de local (S), classe de dap (CLA), índice de competição independente da distância $\left(\mathrm{IID}_{\mathrm{i}}\right)$, dap na idade atual $\left(\right.$ dap $\left._{1}\right)$, altura total na idade atual $\left(\mathrm{h}_{1}\right)$. As variáveis de saída foram: a probabilidade de mortalidade (PM); dap na idade futura $\left(\mathrm{dap}_{2}\right)$; e altura total das árvores na idade futura $\left(\mathrm{h}_{2}\right)$.

Foram testados cinco IID $_{\mathrm{i}}$ (Martins et al, 2011): $\mathrm{IID}_{\mathrm{i}}=\mathrm{dap}_{\mathrm{i}}^{2} / \overline{\mathrm{D}}^{2} ; \mathrm{IID}_{2}=\mathrm{h}_{\mathrm{i}} / \overline{\mathrm{H}} ; \mathrm{IID}_{3}=\left(\operatorname{dap}_{\mathrm{i}}^{2} \times \mathrm{h}_{\mathrm{i}}\right) /\left(\overline{\mathrm{D}}^{2} \times \overline{\mathrm{H}}\right)$; $\mathrm{IID}_{4}=$ dap $_{\mathrm{i}}^{2} / \mathrm{q}^{2} ;$ e $\mathrm{IID}_{5}=\mathrm{BAL}_{\mathrm{i}}$, em que: dap é o dap da árvore-objeto; $\overline{\mathrm{D}}$, a média aritmética dos diâmetros das árvores da parcela; $\mathrm{h}_{\mathrm{i}}$, a altura total da árvore-objeto; $\overline{\mathrm{H}}$, a altura total média das árvores da parcela; q, o diâmetro 
quadrático; e $\mathrm{BAL}_{\mathrm{i}}$, o somatório das áreas seccionais das árvores maiores que a árvore-objeto.

A probabilidade de mortalidade por classe de dap foi obtida pela expressão $P M=100\left(n_{j 1}-n_{j 2}\right) / \sum_{i=1}^{j} n_{j 1}$ (Martins et al., 2011), em que: $\mathrm{n}_{\mathrm{j} 1}$ é o número de árvores vivas na j-ésima classe de diâmetro, no início do período; e $n_{\mathrm{j} 2}$ é o número de árvores vivas na j-ésima classe de diâmetro no fim do período.

Foram treinadas: 500 redes para a estimativa da probabilidade de morte das árvores, com quatro variáveis de entrada $\left(\mathrm{I}_{1}, \mathrm{~S}, \mathrm{CLA}, \mathrm{IID}_{\mathrm{i}}\right) ; 500$ redes para a variável $\mathrm{h}_{2}$, com cinco variáveis de entrada $\left(\mathrm{I}_{1}, \mathrm{I}_{2}, \mathrm{~S}, \mathrm{Ht}_{1}, \mathrm{IID}_{\mathrm{i}}\right)$; e 500 redes para a variável dap ${ }_{2}$, também com cinco variáveis de entrada $\left(\mathrm{I}_{1}, \mathrm{I}_{2}, \mathrm{~S}\right.$, dap $\left._{1}, \mathrm{IID}_{\mathrm{i}}\right)$.

A seleção das cinco melhores redes, para cada variável de saída, baseou-se no coeficiente de correlação entre os valores observados e estimados e no coeficiente de variação (Campos \& Leite, 2009).

A validação do MPT foi realizada pela projeção das áreas basais e dos volumes das 30 parcelas permanentes na idade inicial (24 meses) para as idades futuras, em incrementos de 12 meses (36, 48, 60 e 72 meses), tendo- se considerado três classes de produtividade $(\mathrm{S}=32, \mathrm{~S}=26$ e $\mathrm{S}=20$ ).

A validação do MAI foi realizada com base no fluxograma de passos básicos e decisões, adotado por Castro (2011) e Martins (2011) (Figura 1), nas três classes de produtividade. A regra adotada para a retirada das árvores que deveriam morrer foi a mesma utilizada por Pretzsch et al. (2002). Para isso, comparou-se a probabilidade de morte estimada com um número aleatório $(\mathrm{Pa})$ entre 0 e 1 . Assim, se $\mathrm{PM}>\mathrm{Pa}$, a árvore morre e deve ser retirada da lista de árvores, caso contrário, a árvore permanece viva e suas dimensões devem ser projetadas para a idade futura. Não foi considerada a possibilidade de ingresso de novas árvores.

Para a seleção do melhor modelo (MPT ou MAI), que oferecesse a estimativa mais precisa da produção volumétrica de madeira, foram elaborados histogramas com os valores observados de volume por hectare e com os valores estimados pelos dois modelos, em cada idade e nas três classes produtividade. Além disso, aplicou-se o teste F de Graybill (Graybill, 1976), para

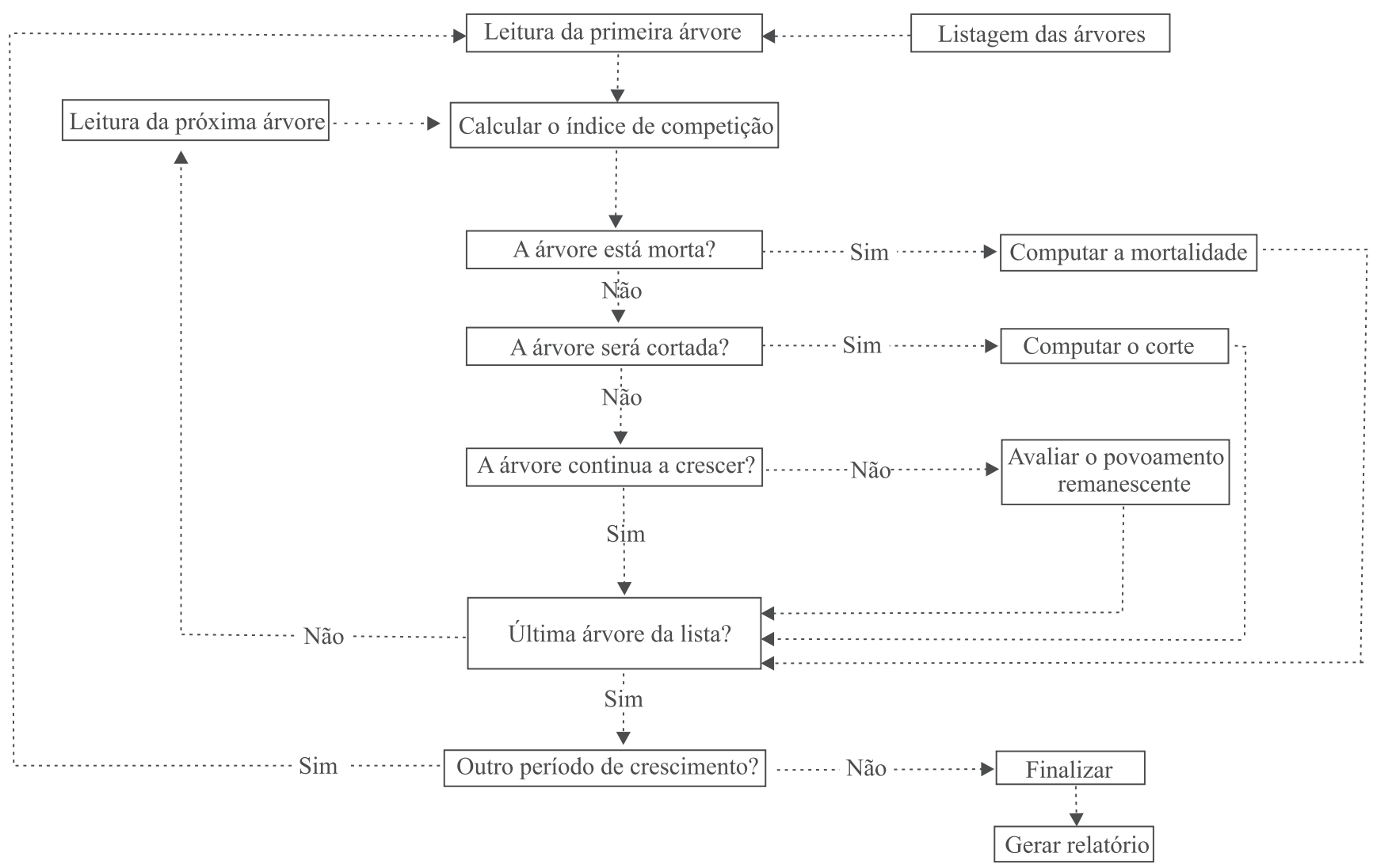

Figura 1. Fluxograma para utilização de um modelo para árvores individuais. 
verificar se as diferenças percentuais entre os volumes estimados pelo MPT e MAI foram estatisticamente diferentes dos volumes observados para cada classe de produtividade (Martins, 2011). Considerou-se a probabilidade de $5 \%$.

\section{Resultados e Discussão}

Com relação ao ajuste do MPT, baseado no sistema de equações simultâneas proposto por Clutter (1963), todos os coeficientes foram estatisticamente significativos pelo teste $\mathrm{t}(\mathrm{p}<0,05)$. Além disso, as equações de área basal e volume ajustaram-se bem aos dados observados, em razão da alta correlação entre os valores observados e estimados e o baixo valor do erro-padrão da estimativa (Tabela 3 ). O sistema de equações simultâneas relaciona os coeficientes aos atributos do povoamento - como idade, índice de local e área basal do povoamento -, e os sinais associados a esses coeficientes devem ser analisados com cuidado, pois apresentam significado biológico (Clutter et al., 1963). No presente estudo, os sinais associados aos coeficientes estão coerentes com a literatura (Campos \& Leite, 2009), em que o coeficiente $\alpha_{1}$, associado à idade, é positivo, e o coeficiente $\beta_{2}$, associado ao inverso da idade, é negativo.

$\mathrm{O}$ modelo de Clutter conseguiu estimar com precisão as áreas basais e os volumes futuros $\left(\mathrm{B}_{2} \mathrm{e} \mathrm{V}_{2}\right)$. No entanto, houve pequena tendência à superestimação das áreas basais menores e subestimação das áreas basais maiores (Figura 2). Essa tendência também foi observada por Dias et al. (2005) e Demolinari (2006) e pode ter ocorrido em consequência das mudanças no povoamento, como estagnação do crescimento em área basal, na idade final da projeção (72 meses). Contudo, esta tendência não influenciou as estimativas

Tabela 3. Estimativas dos coeficientes para área basal e volume e estatísticas dos ajustes dos modelos para povoamento total (MPT), pelas equações simultâneas de Clutter (1936).

\begin{tabular}{|c|c|c|c|c|c|c|}
\hline \multirow[t]{2}{*}{ Parâmetro } & \multicolumn{2}{|c|}{ Área basal } & \multicolumn{4}{|c|}{ Volume } \\
\hline & $\alpha_{1}$ & $\alpha_{2}$ & $\beta_{1}$ & $\beta_{2}$ & $\beta_{3}$ & $\beta_{4}$ \\
\hline Estimativa & 2,4189 & 0,0398 & 1,7494 & $-5,2798$ & 0,0170 & 1,1166 \\
\hline Teste $\mathrm{t}$ & 34,6029 & 10,6879 & 24,5102 & $-1,7951$ & 4,7795 & 28,0279 \\
\hline Significância & 0,0000 & 0,0000 & 0,0000 & 0,0438 & 0,0000 & 0,0000 \\
\hline Correlação & \multicolumn{2}{|c|}{0,9674} & \multicolumn{4}{|c|}{0,9815} \\
\hline Erro-padrão (\%) & \multicolumn{2}{|c|}{ $\pm 6,2$} & \multicolumn{4}{|c|}{ $\pm 6,7$} \\
\hline
\end{tabular}
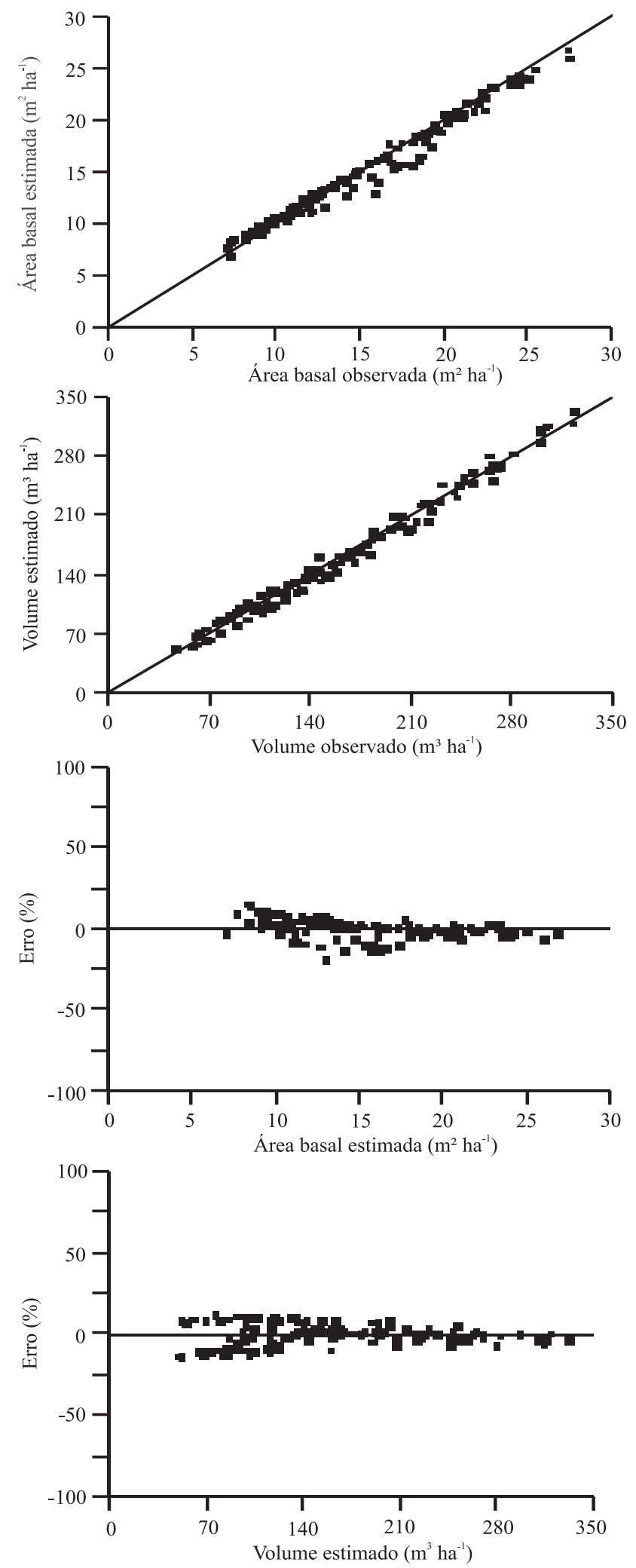

Figura 2. Relação entre valores observados e estimados de área basal e volume; e percentagem de erro das estimativas do modelo para povoamento total. 
de $V_{2}$ que, de maneira geral, estiveram distribuídas próximas à linha $1: 1$, sem que tenha havido tendência de superestimação ou subestimação.

Outros trabalhos relataram dados semelhantes, com bom ajuste do modelo de Clutter (1963) aos dados observados em plantios de Pinus (Leite et al., 2001) e de eucalipto não desbastado (Soares et al., 2004; Demolinari et al., 2007) e desbastado (Dias et al., 2005; Görgens et al., 2007).

Com relação ao MAI, as melhores arquiteturas de redes neurais encontradas para estimar as variáveis de saída (PM, $\mathrm{h}_{2}$, dap $\mathrm{p}_{2}$ ) foram: $\mathrm{M}_{3}$, para $\mathrm{PM} ; \mathrm{H}_{4}$, para $\mathrm{h}_{2}$, e

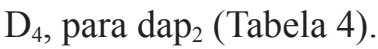

A variável de saída probabilidade de morte (PM) é considerada de difícil estimação, por se tratar de um evento relativamente raro, aleatório e com causas não inteiramente identificadas no povoamento (Martins, 2001; Crecente-Campo et al., 2009). No entanto, essa variável apresentou boas estimativas com o uso das redes neurais artificiais, com altos valores de correlação e baixos valores de CV. Os valores encontrados para a PM (Tabela 4), bem como a proximidade desses valores à linha 1:1 (Figura 3), foram superiores aos encontrados com técnicas de regressão tradicional, o que também

Tabela 4. Arquitetura das cinco redes neurais artificiais de melhor arquitetura $\left(\mathrm{M}_{1}-\mathrm{M}_{5}\right)$, treinadas quanto à probabilidade de mortalidade, altura e diâmetro (dap) em idades futuras $\left(\mathrm{I}_{2}\right)$, com respectivas estatísticas.

\begin{tabular}{|c|c|c|c|c|c|c|}
\hline \multirow[t]{2}{*}{ Rede } & \multirow{2}{*}{$\begin{array}{c}\text { Variável de } \\
\text { entrada }\end{array}$} & \multicolumn{3}{|c|}{ № de neurônios por camada } & \multirow[t]{2}{*}{$\mathrm{r}$} & \multirow{2}{*}{$\begin{array}{l}\text { CV } \\
(\%)\end{array}$} \\
\hline & & Entrada & Oculta & Saída & & \\
\hline & \multicolumn{6}{|c|}{ Probabilidade de mortalidade } \\
\hline $\mathrm{M}_{1}$ & $\mathrm{I}_{1}, \mathrm{~S}, \mathrm{CLA}, \mathrm{IID}_{1}$ & 4 & 9 & 1 & 0,744 & 43,1 \\
\hline $\mathrm{M}_{2}$ & $\mathrm{IID}_{2}$ & 1 & 3 & 1 & 0,579 & 52,3 \\
\hline $\mathrm{M}_{3}$ & $\mathrm{I}_{1}, \mathrm{~S}, \mathrm{CLA}, \mathrm{IID}_{3}$ & 4 & 8 & 1 & 0,799 & 38,6 \\
\hline $\mathrm{M}_{4}$ & $\mathrm{I}_{1}, \mathrm{~S}, \mathrm{CLA}, \mathrm{IID}_{4}$ & 4 & 5 & 1 & 0,711 & 45,2 \\
\hline \multirow[t]{2}{*}{$\mathrm{M}_{5}$} & $\mathrm{I}_{1}, \mathrm{CLA}, \mathrm{IID}_{5}$ & 3 & 4 & 1 & 0,790 & 39,3 \\
\hline & \multicolumn{6}{|c|}{ Altura $\left(\mathrm{h}_{2}\right)$} \\
\hline $\mathrm{H}_{1}$ & $\mathrm{~S}, \mathrm{~h}_{1}, \mathrm{IID}_{1}$ & 3 & 6 & 1 & 0,993 & 3,0 \\
\hline $\mathrm{H}_{2}$ & $\mathrm{I}_{1}, \mathrm{I}_{2}, \mathrm{~S}, \mathrm{~h}_{1}, \mathrm{IID}_{2}$ & 5 & 6 & 1 & 0,993 & 2,9 \\
\hline $\mathrm{H}_{3}$ & $\mathrm{I}_{1}, \mathrm{I}_{2}, \mathrm{~S}, \mathrm{~h}_{1}, \mathrm{IID}_{3}$ & 5 & 7 & 1 & 0,994 & 2,6 \\
\hline $\mathrm{H}_{4}$ & $\mathrm{I}_{1}, \mathrm{I}_{2}, \mathrm{~S}, \mathrm{~h}_{1}, \mathrm{IID}_{4}$ & 5 & 7 & 1 & 0,995 & 2,5 \\
\hline \multirow[t]{2}{*}{$\underline{\mathrm{H}_{5}}$} & $\mathrm{I}_{1}, \mathrm{I}_{2}, \mathrm{~S}, \mathrm{~h}_{1}, \mathrm{IID}_{5}$ & 5 & 9 & 1 & 0,993 & 2,9 \\
\hline & \multicolumn{6}{|c|}{ Diâmetro $\left(\mathrm{dap}_{2}\right)$} \\
\hline $\mathrm{D}_{1}$ & $\mathrm{I}_{1}, \mathrm{I}_{2}, \operatorname{dap}_{1}, \mathrm{IID}_{1}$ & 4 & 5 & 1 & 0,990 & 4,6 \\
\hline $\mathrm{D}_{2}$ & $\mathrm{I}_{1}, \mathrm{I}_{2}, \mathrm{~S}, \mathrm{dap}_{1}, \mathrm{IID}_{2}$ & 5 & 7 & 1 & 0,990 & 4,4 \\
\hline $\mathrm{D}_{3}$ & $\mathrm{I}_{2}, \operatorname{dap}_{1}, \mathrm{IID}_{3}$ & 3 & 4 & 1 & 0,993 & 3,8 \\
\hline $\mathrm{D}_{4}$ & $\mathrm{I}_{1}, \mathrm{I}_{2}, \mathrm{~S}, \mathrm{dap}_{1}, \mathrm{IID}_{4}$ & 5 & 5 & 1 & 0,993 & 3,7 \\
\hline $\mathrm{D}_{5}$ & $\mathrm{I}_{1}, \mathrm{I}_{2}, \mathrm{~S}$, dap $_{1}, \mathrm{IID}_{5}$ & 5 & 6 & 1 & 0,988 & 4,9 \\
\hline
\end{tabular}

Pesq. agropec. bras., Brasília, v.48, n.3, p.287-295, mar. 2013 DOI: 10.1590/S0100-204X2013000300007 foi observado em abeto-branco (Picea glauca) por Yang et al. (2003), em acácia-negra por Schneider et al. (2005), e em eucalipto por Martins (2011). As variáveis de entrada para PM (I, S, CLA e o índice de competição $\mathrm{IID}_{3}$ ) são biologicamente consistentes, pois, juntas representam o status competitivo de cada árvore, que está associado à mortalidade (Martins et al., 2011).

As variáveis de saída $\mathrm{h}_{2}$ e dap $\mathrm{p}_{2}$ também apresentaram excelentes estimativas com o uso das redes neurais, com valores próximos à linha 1:1 (Figura 3), o que justifica sua utilização em substituição aos tradicionais modelos de regressão. As redes neurais não apresentam uma estrutura definida, como é o caso dos modelos de regressão. Elas têm a capacidade de modelar aspectos não lineares sem a prévia definição de uma função, o que economiza tempo e esforço, na escolha de modelos de regressão, e facilita a estimativa de um grande número de arquiteturas pelos programas especializados. Além disso, as variáveis de entrada para $\mathrm{h}_{2}$ e dap $\operatorname{di}_{2}\left(\mathrm{I}_{1}, \mathrm{~S}, \mathrm{~S}\right.$, índice de competição IID4 e os valores de $h_{1}$ e dap ${ }_{1}$ ) são amplamente utilizadas na projeção da altura e diâmetro em plantios florestais (Campos \& Leite, 2009). No presente trabalho, essas variáveis propiciaram maior precisão do que a obtida por Binoti (2010) em plantios de eucalipto na mesma faixa de idade.

Na validação de MPT e de MAI, as estimativas dos volumes de madeira por hectare, obtidas pelo MPT, apresentaram menor desvio-padrão do que os observados com uso do MAI, nas três classes de produtividade (Figura 4 ).

Na classe $S=32$, nas idades iniciais (36 e 48 meses), o volume projetado pelo MAI foi mais próximo aos volumes observados. Nas idades finais (60 e 72 meses), o volume projetado pelo MPT foi mais próximo dos valores observados. Nas classes $S=26$ e $S=20$, em todas as idades, o MAI propiciou estimativa mais precisa. Além disso, o MPT subestimou os volumes na classe $\mathrm{S}=26$ e, em $\mathrm{S}=20$, os superestimou em todas as idades.

As diferenças dos volumes de madeira estimados e observados e os estimados nas classes $S=32$ foram significativas nos dois modelos: no caso do MAI, aos 60 meses; no caso do MPT, aos 36 e 48 meses (Tabela 5). No MPT, houve tendência de subestimação dos volumes nas idades iniciais, o que pode ter ocorrido em razão da deficiência no ajuste do modelo 
de Clutter para $\mathrm{B}_{2}$ (Figura 2) e pode ter inflacionado o volume estimado nessas idades. Na classe $S=26$, todas as diferenças nos volumes estimados e observados com o MAI não foram significativas, o que mostra a superioridade deste modelo em comparação ao MPT nessa classe. $\mathrm{Na} S=20$, o MAI também foi mais preciso do que o MPT, mesmo com a diferença significativa observada aos 72 meses.
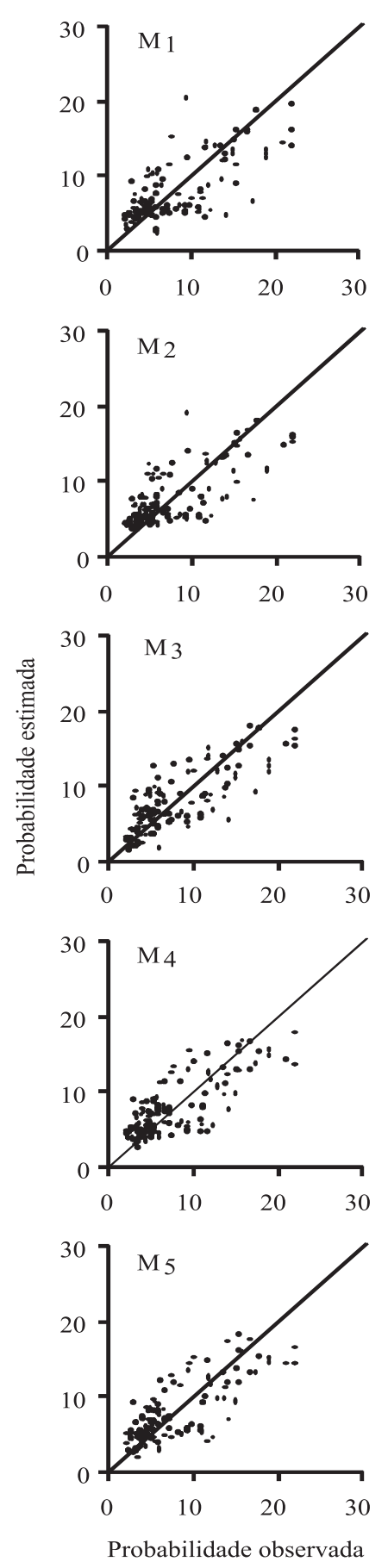
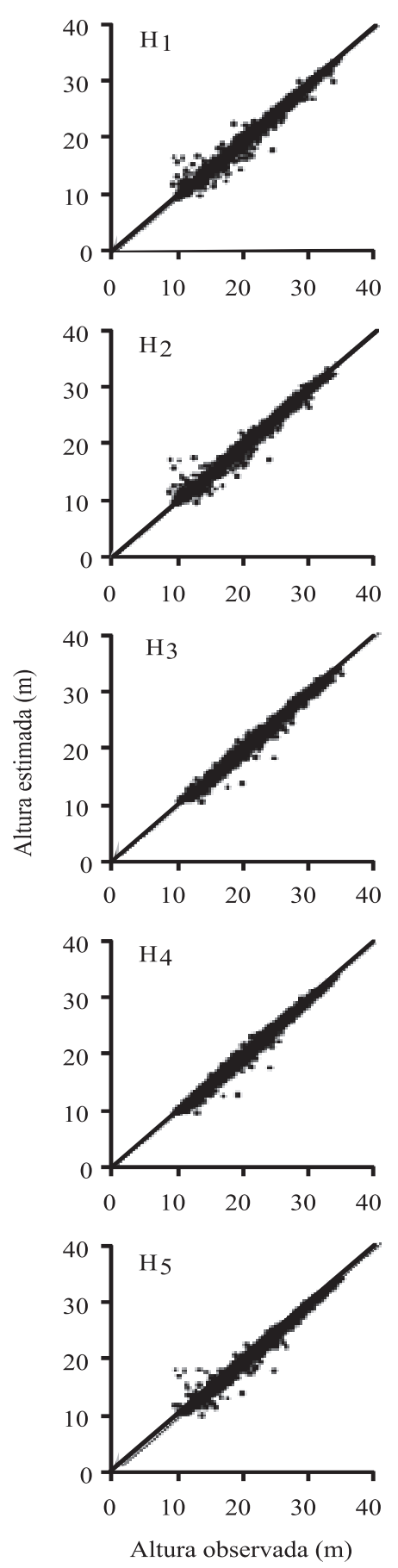
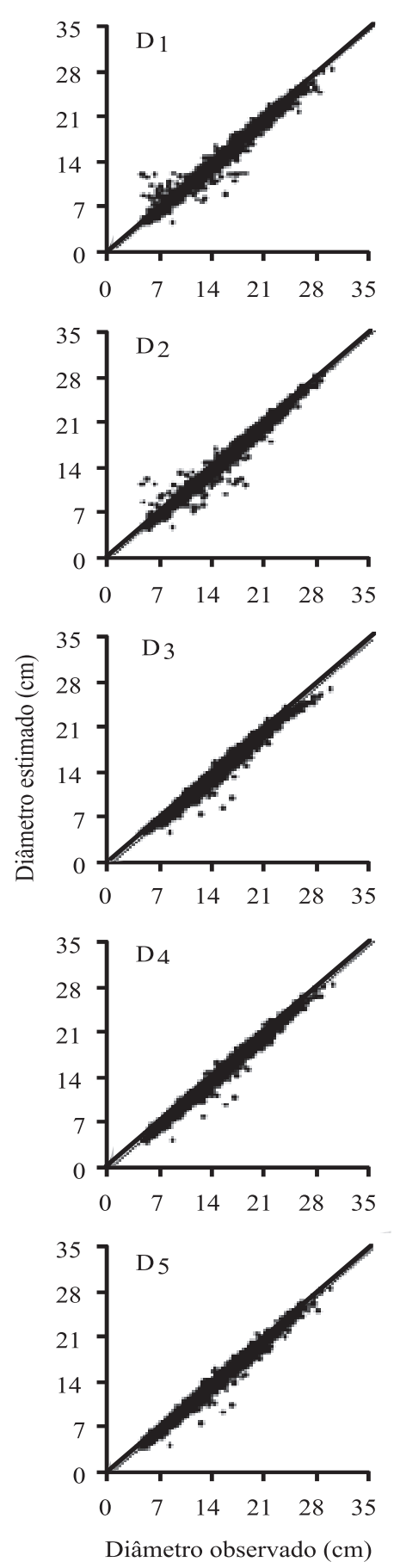

Figura 3. Probabilidade de mortalidade, altura e diâmetro, observados e estimados pelas cinco melhores redes neurais artificiais $\left(\mathrm{M}_{1}\right.$ à $\mathrm{M}_{5}, \mathrm{H}_{1}$ à $\mathrm{H}_{5}$ e $\mathrm{D}_{1}$ à $\left.\mathrm{D}_{5}\right)$. 

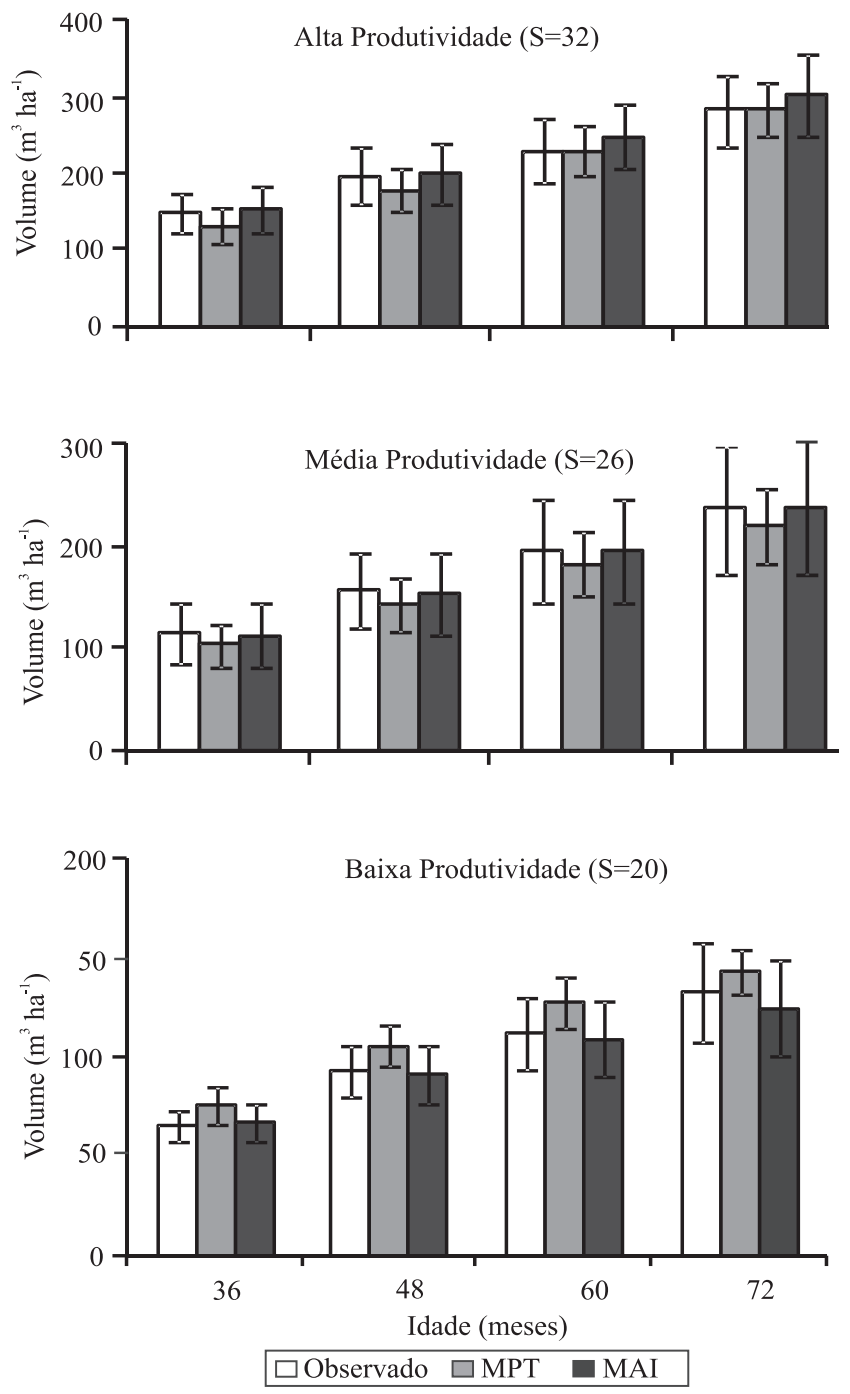

Figura 4. Volume de madeira observado e estimado pelos modelos para povoamento total (MPT) e para árvore individual (MAI), em função da idade e de cada classe de produtividade (as barras representam o desvio-padrão). S, índice de local.

Tabela 5. Diferença percentual entre os volumes de madeira $\left(\mathrm{m}^{3} \mathrm{ha}^{-1}\right)$ observados e estimados pelos modelos para povoamento total (MPT) e para árvore individual (MAI), em diferentes classes de produtividade ( $\mathrm{S}=20,26$ e 32).

\begin{tabular}{|c|c|c|c|c|c|c|}
\hline \multirow{2}{*}{$\begin{array}{l}\text { Idade } \\
\text { (meses) }\end{array}$} & \multicolumn{2}{|c|}{$\mathrm{S}=32$} & \multicolumn{2}{|c|}{$\mathrm{S}=26$} & \multicolumn{2}{|c|}{$\mathrm{S}=20$} \\
\hline & MPT & MAI & MPT & MAI & MPT & MAI \\
\hline 36 & $-12^{*}$ & $2^{\mathrm{ns}}$ & $-10^{*}$ & $-2^{\mathrm{ns}}$ & $15^{*}$ & $2^{\mathrm{ns}}$ \\
\hline 48 & $-9 *$ & $1^{\text {ns }}$ & $-9 *$ & $-1^{\mathrm{ns}}$ & $15^{*}$ & $-1^{\mathrm{ns}}$ \\
\hline 60 & $0^{\text {ns }}$ & $8^{*}$ & $-7 *$ & $0^{\mathrm{ns}}$ & $13^{*}$ & $-3^{\text {ns }}$ \\
\hline 72 & $1^{\text {ns }}$ & $7^{\text {ns }}$ & $-6^{*}$ & $0^{\text {ns }}$ & $8^{*}$ & $6^{*}$ \\
\hline
\end{tabular}

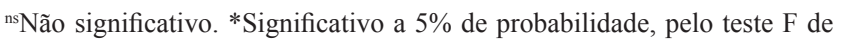
Graybill (1976). S, índice de local.
Ambos os modelos foram adequados para estimar o volume de madeira por hectare. As prognoses garantiram estimativas precisas em todas as idades, principalmente nas idades finais. No entanto, o MAI foi o mais preciso em quase todas as idades e classes de produtividade, exceto para a $\mathrm{S}=36$ nas idades de 60 e 72 meses (Figura 4 e Tabela 5). Assim, constatase que esse modelo pode ser um importante método para a prognose no manejo florestal de Eucalyptus. Neste sentido, o recurso das redes neurais artificiais pode servir como alternativa às funções que compõem o MAI e auxiliar na identificação das variáveis mais importantes, para predizer o crescimento em diâmetro, altura e probabilidade de mortalidade em árvores individuais, bem com a produção volumétrica de um povoamento.

\section{Conclusões}

1. O ajuste do modelo para povoamento total, pelo sistema de equações simultâneas de Clutter, e o ajuste do modelo para árvore individual, pelas redes neurais artificiais, são consistentes.

2. O modelo para árvore individual é mais preciso na estimativa do volume de madeira por hectare, em plantios comerciais de eucalipto.

\section{Referências}

ASSMANN, E. The principles of forest yield study. New York: Pergamon, 1970. 506p.

BINOTI, M.L.M.S. Redes neurais artificiais para prognose da produção de povoamentos não desbastados de eucalipto. 2010. 54p. Dissertação (Mestrado) - Universidade Federal de Viçosa, Viçosa.

CAMPOS, J.C.C.; LEITE, H.G. Mensuração florestal: perguntas e respostas. 3.ed. Viçosa: UFV, 2009. 548p.

CASTRO, R.V.O. Modelagem do crescimento em nível de árvores individuais utilizando redes neurais e autômatos celulares. 2011. 80p. Dissertação (Mestrado) - Universidade Federal de Viçosa, Viçosa.

CLUTTER, J.L. Compatible growth and yield models for loblolly pine. Forest Science, v.9, p.354-371, 1963.

CRECENTE-CAMPO, F.; MARSHALL, P.; RODRÍGUEZ-SOALLEIRO, R. Modeling non-catastrophic individual tree mortality for Pinus radiate plantations in Northwestern Spain. Forest Ecology and Management, v.257, p.1542-1550, 2009. DOI: 10.1016/j.foreco.2009.01.007.

DAVIS, L.S.; JOHNSON, K.N.; BETTINGER, P.; HOWARD, T.E. Forest management: to sustain ecological, economic, and social values. 4.ed. Illinois: Waveland, 2005. 804p. 
DEMOLINARI, R. de A. Crescimento de povoamentos de eucalipto não desbastados. 2006. 72p. Dissertação (Mestrado) Universidade Federal de Viçosa, Viçosa.

DEMOLINARI, R. de A.; SOARES, C.P.B.; LEITE, H.G.; SOUZA, A.L. de. Crescimento de plantios clonais de eucalipto não desbastados na região de Monte Dourado (PA). Revista Árvore, v.31 p.503-512, 2007. DOI: 10.1590/S0100-67622007000300016.

DIAS A.N.; LEITE, H.G.; CAMPOS, J.C.C.; COUTO, L.; CARVALHO, A.F. de. Emprego de um modelo de crescimento e produção em povoamentos desbastados de eucalipto. Revista Árvore, v.29, p.731-739, 2005. DOI: 10.1590/ S0100-67622005000500008.

GONZÁLEZ, M.S.; RÍO, M. del; CAÑELLAS, I.; MONTERO, G. Distance independent tree diameter growth model for cork oak stands. Forest Ecology and Management, v.225, p.262-270, 2006. DOI: 10.1016/j.foreco.2006.01.002.

GÖRGENS, E.; LEITE, H.G.; NOGUEIRA, G.S. ; DIAS, A.N. Tendência de crescimento de povoamento de eucalipto após aplicação de desbaste. Revista Árvore, v.31, p.879-885, 2007. DOI: 10.1590/S0100-67622007000500012.

GRAYBILL, F.A. Theory and application of linear model. North Scituate: Duscbiry, 1976. 704p.

HASENAUER, H. Sustainable forest management: growth models for Europe. Berlin: Springer, 2006. 398p. DOI: 10.1007/3-540-31304-4.

HAYKIN, S. Redes neurais: princípios e prática. 2.ed. Porto Alegre: Bookman, 2001. 900p.

IHS GLOBAL. Eviews 5: quantitative micro software. Version 5. Irvine: IHS Global, 2004.

LEITE, H.G.; NOGUEIRA, G.S.; MOREIRA, A.M.; LIMA, J.E. de. Um modelo de crescimento e produção para Pinus taeda L. na região Sul do Brasil. Revista Árvore, v.25, p.105-112, 2001.

LEITE, H.G.; OLIVEIRA-NETO, R.R. de; MONTE, M.A.; FARDIN, L.; ALCANTARA, A.M. de; BINOTI, M.L.M. da S.; CASTRO, R.V.O. Modelo de afilamento de cerne de Tectona grandis L.f. Scientia Forestalis, v.39, p.53-59, 2011.

MABVURIRA, D.; MIINA, J. Individual tree growth and mortality models for Eucalyptus grandis (Hill) Maiden plantations in Zimbabwe. Forest Ecology and Management, v.161, p.231-245, 2002. DOI: $10.1016 / \mathrm{S} 0378-1127(01) 00494-7$.
MARTINS, F.B. Modelagem de crescimento em nível de árvore individual para plantios comerciais de eucalipto. 2011. 143p. Tese (Doutorado) - Universidade Federal de Viçosa, Viçosa.

MARTINS, F.B.; SOARES, C.P.B.; LEITE, H.G.; SOUZA, A.L. de; CASTRO, R.V.O. Índices de competição em árvores individuais de eucalipto. Pesquisa Agropecuária Brasileira, v.46, p.1089-1098, 2011. DOI: 10.1590/S0100-204X2011000900017.

MIGUEL, E.P.; MACHADO, S. do A.; FIGUEIREDO FILHO, A.; ARCE, J.E. Using the Weibull function for prognosis of yield by diameter class in Eucalyptus urophylla stands. Cerne, v.16, p.94-104, 2010.

MONTY, A.; LEJEUNE, P.; RONDEUX, J. Individual distance-independent girth increment model for Douglas-fir in southern Belgium. Ecological Modelling, v.212, p.472-479, 2008. DOI: 10.1016/j.ecolmodel.2007.10.041.

PRETZSCH, H.; BIBER, P.; ĎURSKÝ, J. The single tree-based stand simulator SILVA: construction, application and evaluation. Forest Ecology and Management, v.162, p.3-21, 2002. DOI: 10.1016/S0378-1127(02)00047-6.

SCHNEIDER, P.R.; FORTES, F. de O.; SILVA, L.H. de S.; LÚCIO, A.D.; FINGER, C.A.G.; PIGATTO, P.S.S. Análise da mortalidade de Acacia mearnsii De Wild. Ciência Florestal, v.15, p.137-143, 2005.

SILVA, M.L.M. da; BINOTTI, D.H.B.; GLERIANI, J.M.; LEITE, H.G. Ajuste do modelo de Schumacher e Hall e aplicação de redes neurais artificiais para estimar volume de árvores de eucalipto. Revista Árvore, v.33, p.1133-1139, 2009. DOI: 10.1590/ S0100-67622009000600015.

SOARES, C.P.B.; LEITE, H.G.; OLIVEIRA, M.L.R. de; CARVALHO, A. Especificação de um modelo de crescimento e produção florestal. Revista Árvore, v.28, p.831-837, 2004. DOI: 10.1590/S0100-67622004000600007.

VANCLAY, J.K. Modelling forest growth and yield: applications to mixed tropical forests. Copenhagen: CAB International, 1994. $312 \mathrm{p}$.

VOSPERNIK, S.; MONSERUD, R.A.; STERBA, H. Do individual tree growth models correctly represent height: diameter ratios of Norway spruce and scots pine? Forest Ecology and Management, v.260, p.1735-1753, 2010. DOI: 10.1016/j.foreco.2010.07.055.

YANG, Y.; TITUS, S.J.; HUANG, S. Modeling individual tree mortality for white spruce in Alberta. Ecological Modelling, v.163, p.209-222, 2003. DOI: 10.1016/S0304-800(03)00008-5.

Recebido em 10 de junho de 2012 e aprovado em 28 de fevereiro de 2013

Pesq. agropec. bras., Brasília, v.48, n.3, p.287-295, mar. 2013 DOI: $10.1590 / \mathrm{S} 0100-204 \mathrm{X} 2013000300007$ 